proper representations. Inverting this procedure, Professor Bell, in his second paper, proves formulas of remarkable simplicity for the expression of the proper number in terms of the total, so that if the latter is within reach of analysis, so also now is the former. A few illustrations are given in the derivation of new results concerning $6,8,10$ or 12 squares and other simple quadratic forms. The cases of 10,12 squares present some unexpected singularities.

7. From 1860 to 1864 Liouville published in his Journal numerous theorems on the number of total and proper representations of integers in special quadratic forms of four and six indeterminates. His formulas for total numbers of representations were for the most part proved in 1890 by Pepin, those omitted being readily demonstrable by elliptic functions and other algebraic means. The formulas for proper representations have not hitherto been proved. Modifying the general principles of his second paper to fit Liouville's forms, Professor Bell demonstrates all of the unproved results very simply. The paper will appear in the Journal de Mathématiques pures et appliquées, (in French), and a fuller abstract shortly in the Paris Comptes Rendus.

8. Professor Winger's paper appeared in full in the November BuLLETIN.

B. A. Bernstein, Secretary of the Section.

\title{
ON THE PROOF OF CAUCHY'S INTEGRAL FORMULA BY MEANS OF GREEN'S FORMULA.
}

BY MR. J. L. WALSH.

(Read before the American Mathematical Society December 30, 1919.)

IT is well known. that Cauchy's integral formula for an analytic function $f(z)=u(x, y)+i v(x, y)$ of the complex variable $z=x+i y$ is analogous to Green's formula for the functions $u$ and $v$, and that moreover Cauchy's formula can be proved from Green's formula. Picard (Traité d'Analyse (1905), volume II, page 114) gives this proof assuming that 
$u$ and $v$ have continuous second partial derivatives, which fact is proved later by means of Cauchy's formula. It is the purpose of the present note to give a modification of Picard's proof which shall not be open to this objection.*

Two functions $U(x, y)$ and $U^{\prime}(x, y)$ are said to be conjugate in a region $T$ of the $x, y$-plane if within that region they are continuous with their first partial derivatives and if in addition

$$
\frac{\partial U}{\partial x}=\frac{\partial U^{\prime}}{\partial y}, \quad \frac{\partial U}{\partial y}=-\frac{\partial U^{\prime}}{\partial x} .
$$

A function $V(x, y)$ is said to be harmonic in $T$ if in $T$ it is continuous together with its first and second partial derivatives and if it satisfies Laplace's equation

$$
\frac{\partial^{2} V}{\partial x^{2}}+\frac{\partial^{2} V}{\partial y^{2}}=0 .
$$

Suppose $U$ and $U^{\prime}$ are conjugate in a region $T$, and that $V$ and $V^{\prime}$ are not only conjugate but also harmonic in $T$. Then if $C$ is any contour bounding a region wholly in $T$, we shall have

$$
\int_{c}\left[U \frac{\partial V}{\partial n}-V \frac{\partial U}{\partial n}\right] d s=0 .
$$

For the analysis given by Picard (l. c., page 10) establishes the following relations, where the double integrals are extended over the interior of $C$ :

$$
\begin{gathered}
\iint\left[\frac{\partial U}{\partial x} \frac{\partial V}{\partial x}+\frac{\partial U}{\partial y} \frac{\partial V}{\partial y}\right] d x d y=-\int_{c} U \frac{\partial V}{\partial n} d s \\
\iint\left[\frac{\partial U^{\prime}}{\partial x} \frac{\partial V^{\prime}}{\partial x}+\frac{\partial U^{\prime}}{\partial y} \frac{\partial V^{\prime}}{\partial y}\right] d x d y=-\int_{c} U^{\prime} \frac{\partial V^{\prime}}{\partial n} d s
\end{gathered}
$$

* Osgood, Funktionentheorie (1912), p. 679, gives such a proof due to Morera, but requiring the existence of a Green's function for the region. He also points out that Green's formula can be derived from that of Cauchy. A proof for the circle, and hence for any region which can be conformally represented on the circle, is given by Kellogg, this BuLletiN, vol. 10 (1903-04), p. 255 .

Compare also J. L. Walsh, "Note on Cauchy's integral formula," Annals of Mathematics, vol. 18, p. 79, where Cauchy's formula is proved by means of Cauchy's integral theorem and a proof of the mean value theorem for harmonic (or conjugate) functions due to Bôcher. 
But by virtue of (1) and the corresponding relations between $V$ and $V^{\prime}$, the left-hand members of the two equations (3) are identical. Hence

$$
\int_{c} U \frac{\partial V}{\partial n} d s=\int_{c} U^{\prime} \frac{\partial V^{\prime}}{\partial n} d s .
$$

Also

$$
\begin{aligned}
0 & =\int_{c} \frac{\partial\left(U^{\prime} V\right)}{\partial s} d s=\int_{c}\left[U^{\prime} \frac{\partial V}{\partial s}+V \frac{\partial U^{\prime}}{\partial s}\right] d s \\
& =\int_{c} U^{\prime} \frac{\partial V^{\prime}}{\partial n} d s-\int_{c} V \frac{\partial U}{\partial n} d s .
\end{aligned}
$$

This immediately gives us equation (2).

Having proved this theorem,* we can continue by means of the analysis of Picard (l. c., page 15), setting $V=\log r$ and proving

$$
U(a, b)=\frac{1}{2 \pi} \int_{c}\left[\log r \frac{\partial U}{\partial n}-U \frac{\partial \log r}{\partial n}\right] d s .
$$

Further developments (l. c., page 114) give us easily Cauchy's integral formula

$$
f\left(z^{\prime}\right)=\frac{1}{2 \pi i} \int_{c} \frac{f(z)}{z-z^{\prime}} d z .
$$

HaRVARD UnIVERSITY.

* This was proved for a region bounded by a single contour, but there is no real difficulty involved in its later application to the multiply connected region. 